\title{
Erratum to: Regulating Factors in Acid-Sensing Ion Channel 1a Function
}

\author{
Yinghong Wang ${ }^{1} \cdot$ Zaven O'Bryant $^{2} \cdot$ Huan Wang ${ }^{1} \cdot$ Yan Huang $^{1}$
}

Published online: 15 February 2016

(C) Springer Science+Business Media New York 2016

\section{Erratum to: Neurochem Res \\ DOI 10.1007/s11064-015-1768-x}

The author of the article has missed to provide the Grant Number for "The Young top talents Program of Anhui Medical University and Foundation for Distinguished Young Talents in Higher Education of Anhui, China" in the acknowledgment section of the original publication.

The Acknowledgment section is corrected with this erratum.

Acknowledgments This study is supported by Research Fund for the Back-up Candidates of the Academic and Technical Leaders of Anhui, China (No. 2015H040), and the Young top talents Program of Anhui Medical University and Foundation for Distinguished Young Talents in Higher Education of Anhui, China (gxyqZD2016049).

The online version of the original article can be found under doi: 10.1007/s11064-015-1768-x.

Yan Huang

aydhy@126.com

1 School of Pharmacy, Anhui Medical University, Meishan Road, Hefei 230032, Anhui, China

2 Department of Neurobiology, Morehouse School of Medicine, 720 Westview Dr., Atlanta, GA, USA 